\title{
Risk information source preferences in construction workers
}

\author{
Calvin Burns, \\ Department of Human Resource Management, \\ University of Strathclyde \\ Stacey Conchie, \\ Department of Psychology, \\ University of Lancaster
}

\section{Introduction}

Many researchers have investigated the determinants of workers' risk-taking / unsafe behaviours as a way to improve safety management and reduce accidents but there has been a general lack of research about workers' risk information seeking behaviours or their source preferences for risk information. It is important to understand workers' risk perceptions and how they are affected by information from different sources because workers tend to underestimate risks which can increase the likelihood of accidents and injury through unsafe behaviours (Powell, 2007; Zohar and Luria, 2004).

The aim of this study was to investigate risk information source preferences in construction workers. Construction is an appropriate industry to study source preferences for occupational risk information as it is one of the most hazardous industries worldwide. Compared to other industries, the construction industry experiences some of the highest annual rates of workplace deaths, accidents and injuries (European Agency for Safety and Health at Work, 2013a; UK Health \& Safety Executive, 2013a; US Department of Labor BLS, 2013). Part of the reason for these high rates is the unique characteristics of the industry. These include a largely transient, project-based workforce, constant time and cost pressures, and a relatively high number of young, migrant, and subcontracted workers (see Lingard and Rowlinson, 2005 for a further description of the nature of the 
Calvin Burns, Stacey Conchie, (2013) "Risk Information Source Preferences in Construction Workers", Employee Relations, Vol. 36 Iss: 1)

construction industry). Construction workers though usually have immediate or fast access to risk information from supervisors, workmates, and (on some projects and in some countries) safety officers or safety managers (Dingsdag, Biggs, \& Sheahan, 2008; Melià, Mearns, Silva, \& Lima, 2008). Together, these characteristics create climates defined by fragmented safety attitudes and risk perceptions, and high levels of risk-taking amongst some groups.

Proximity to Source of Risk Information

Johnson's (1996) theory of local information fields provides a good starting point for understanding construction workers' source preferences for risk information. According to this theory, local information fields encompass the sources of information to which an individual is exposed on a daily basis. Through regular contact, individuals develop a preference towards these sources following a process of familiarity or normative behaviour. In the construction industry, local information fields would likely comprise supervisors, workmates, and safety managers. Of these different occupational groups, Johnson's theory suggests that supervisors and workmates are likely to be the preferred sources of risk information as these groups engage with workers on a daily basis. Consequently, workers are likely to approach supervisors and workmates for risk information and advice as a matter of routine behaviour.

Immediate or fast access to a source of risk information, however, may not always affect information seeking. Savolainen (2008) demonstrated that individuals can be selective about the source to which they respond irrespective of how quickly they can approach that source. Mearns and Reader (2008) found that workers' safety behaviours were more strongly affected by supervisors than by workmates, even though both sources 
Calvin Burns, Stacey Conchie, (2013) "Risk Information Source Preferences in Construction Workers", Employee Relations, Vol. 36 Iss: 1)

were in the same local information field. Melià et al. (2008) reported similar findings in relation to the Spanish construction industry, where workers' perceptions of risk were influenced more strongly by supervisors' responses to risk, than workmates (but the organizational response was a stronger influence on risk perceptions than supervisors' response). Lastly, Dingsdag et al. (2008) showed that Australian construction workers' safety attitudes were influenced relatively more by supervisors and safety managers than by workmates. These findings have been explained in terms of safety climate (members' shared attitudes and perceptions about risk which inform the role behaviours that are rewarded and supported in the organization; Zohar \& Luria, 2004). They may also reflect workers' source preferences (i.e. the sources that workers are most likely to approach for information and presumably by which their behaviour will be most influenced).

\section{Cross-Cultural Factors}

There are a large number of foreign transient workers in the construction industry who often struggle with the English language (Sinclair et al., 2008) and this may affect their risk information seeking behaviours. Aronsson (1999) found that contingent workers reported a greater lack of work environment knowledge than permanent workers and perceived themselves to be disadvantaged with respect to the training and education needed to do their jobs. Burt et al. (2008) found that foreign transient construction workers often experience difficulties in understanding safety documents which are not translated into their native language unless an interpreter communicates that information. Communicating about and seeking information on occupational risk in the construction industry may thus be made more difficult due to cross-cultural factors. 
Calvin Burns, Stacey Conchie, (2013) "Risk Information Source Preferences in Construction Workers", Employee Relations, Vol. 36 Iss: 1)

The influence of national culture on risk perceptions has been reflected in a growing number of studies (e.g., Helmrich \& Merritt, 1998; Mearns \& Yule, 2009). An example of this within the construction industry comes from Spagenberg, Baarts, Dyreborg et al., (2003), who found that Danish construction workers experienced more accidents than Swedish workers despite both being on the same site. The differences between these groups were accounted for by differences in education, training, organizational commitment, economic interest, and safety attitudes. Spagenberg et al. (2003) concluded that nationality per se does not influence risk perceptions and behaviour, but the factors to which it relates to does. Support for their conclusion was later offered by Mearns and Yule (2009) who provided evidence that national culture is secondary to the influence of organizational culture, especially when the latter is strong. To date the role of cross-cultural factors on risk information seeking behaviours remains largely unexplored.

\section{Trust in Source of Risk Information}

Trust has long been known to affect whether a risk is accepted or not. It is often presented as a psychological state in which one person (trustor) chooses to rely on another person (trustee) in a risky situation based upon positive expectations of the trustee's behaviour or intentions (Rousseau et al., 1998). It is widely accepted that these expectations are domain-specific and that in the context of occupational risk, expectations about another's safety-related behaviours and safety-related intentions are particularly important (Conchie, Donald, \& Taylor 2006). In most cases, a trustor's expectations about a trustee relate to the trustee's trustworthiness, which is indicated through a number of personal qualities (ability, benevolence, integrity; Mayer, Davis, \& Schoorman, 1995). 
Calvin Burns, Stacey Conchie, (2013) "Risk Information Source Preferences in Construction Workers", Employee Relations, Vol. 36 Iss: 1)

In a previous study of UK construction workers, Conchie and Burns (2009) found that the HSE and safety managers were the most trusted sources of occupational risk information (compared to project managers, supervisors, and workmates) and the most influential in shaping workers' risk-related behavioural intentions. They also found that workers' trust in an information source was relatively stable and did not change significantly depending on the occupational risk that the source was communicating about (e.g., workers reported that they trusted the HSE just as much to communicate about back pain as site transport). Trust in risk information from the project manager, safety manager, HSE, and workmates was found to be based on perceptions of the source's accuracy while trust in risk information from supervisors was found to be based on their demonstrations of care. Their findings have implications for safety campaigns because they suggest that while workers trust the sources that develop these campaigns (HSE, and perhaps safety managers), they have relatively less trust (but not necessarily distrust) in those sources that deliver those safety campaign messages (project managers and supervisors). While trust in risk information sources in occupational settings has started to be investigated, the role of source preference for risk information on trust remains unclear.

\section{Current Study}

The aim of this study was to investigate risk information source preferences in construction workers. Previous research about risk information source preferences has tended to focus on industrial / technological hazards and risks amongst the general public (e.g., Jungermann, Pfister, \& Fischer, 1996). To our knowledge, this is the first study to investigate risk information source preferences in an occupational setting. 
Calvin Burns, Stacey Conchie, (2013) "Risk Information Source Preferences in Construction Workers", Employee Relations, Vol. 36 Iss: 1)

As discussed earlier, the information seeking literature suggests that source proximity is an important factor in determining information source preference. Local Information Field theory (Johnson, 1996) predicts that construction workers will show a relatively stronger preference for risk information from proximal sources like supervisors and workmates, than from sources like project managers, safety managers, or government bodies (i.e., UK Health and Safety Executive). Recent findings about construction workers' trust in sources of occupational risk information (Conchie \& Burns, 2009) suggest that source expertise (based on perceptions of accuracy) may also be an important factor in determining risk information source preference. Source expertise has been found to be important for laypeople's source preferences for information about industrial / technological risks. For example, Jungermann et al. (1996) found that residents local to a chemical plant preferred to receive information about hazards and their health consequences from environmentalists, but preferred to receive information about how to act after an accident from the fire department. Their study and others (Frewer et al., 1996; Warner et al., 1973) suggest that proximity alone is an insufficient basis for source preference. Rather, proximity is likely to combine with, or be superseded by, how much expertise a source is assumed to have about a specific risk. Thus, we investigated whether occupational risk information source preference was risk independent (i.e. whether construction workers prefer to receive occupational risk information from proximal sources like supervisors and workmates regardless of the nature of the risk or the source's expertise regarding that risk, or if they discriminated between information sources based on the type of risk being considered). 
Lastly, we sought to extend the methods used to investigate information source preferences. Previous research about risk information source preferences (e.g., Jungermann et al., 1996) has focused only on people's most preferred source. In this study, we examined how workers ranked a source in terms of how much that source was preferred relative to other sources to deliver information about a specific risk. This method allowed for a more detailed picture of risk information source preference than may be gleaned by focusing on the most preferred source only. 
Calvin Burns, Stacey Conchie, (2013) "Risk Information Source Preferences in Construction Workers", Employee Relations, Vol. 36 Iss: 1)

\section{Method}

\section{Participants and Procedure}

We collected data from 106 frontline construction workers who were recruited from a large, single building site within the UK. This study was conducted at the same site as our previous study (Conchie \& Burns, 2009) after data from that study had been collected. Research access was co-ordinated through the on-site safety officer once permission to conduct the study from the School of Psychology's ethics committee at the University of Liverpool was granted. Available workers were selected through opportunity sampling, such that the on-site safety officer identified groups of workers who were available to take part in the study ${ }^{1}$. These workers were approached by the second author and asked to participate in a study on risk communication within the construction industry (if they had not taken part in our previous study). All of the workers who were approached agreed to participate. They were given a participant information sheet which assured them of anonymity and confidentiality along with the ranking exercise. They completed the ranking exercise on-site during working hours and once they completed the ranking exercise, they returned it directly to the second author who was there at the time.

All of the workers were male and collectively were employed by a number of different contracting companies. Together the sample represented a range of trades, with the largest groups being labourers $(25 \%)$, bricklayers (12\%), electricians (11\%), scaffold workers $(9 \%)$, fitters $(7 \%)$, steel and plate fitters $(6 \%)$, and welders $(5 \%)$. Other trades

\footnotetext{
1 'Unavailable' workers were those carrying out tasks that needed to be completed during the time that the study was conducted.
} 
Calvin Burns, Stacey Conchie, (2013) "Risk Information Source Preferences in Construction Workers", Employee Relations, Vol. 36 Iss: 1)

represented by one or two workers included plasters, painters, plumbers and banksmen. Sixteen percent of workers did not disclose their trade.

\section{Ranking Exercise}

The source from which workers preferred to receive information about a range of risks was measured using a ranking exercise. Specifically, workers were asked to rank five occupational sources according to how much they preferred each one to deliver information about eight different risks. A rank of five was given to the source from which workers most preferred to receive risk information, a four to the next preferred source, and so on until they arrived at the least preferred source to which they assigned a rank of one. In doing this, all sources were assigned a rank from " 1 " to " 5 " for each of the eight risks. The procedure of assigning a rank to all information sources, rather than assigning a rank to the most preferred source only, had the advantage of providing information on the relative position of each source in terms of worker preference.

The five sources that workers were asked to rank were the UK HSE, Safety manager, Project manager, Supervisor, and Workmates. These occupational groups have been identified as key agents in shaping workers' attitudes and perceptions toward safety in a range of industries including construction (Cox and Cox, 1991; Hayes et al., 1998; Melià et al., 2008; Seo et al., 2004). The eight risks were Asbestos, Back pain, Site transport, Working at heights, Slips/trips, Bad housekeeping, Site-specific risk (i.e., risks that are specific to the building site surveyed), and Job-specific risk (i.e., risks that are specific to the nature of the worker's job). The first six of these risks are specific risks which have been identified by regulatory agencies to be relevant to the construction industry and in some cases the main risks to which construction workers are exposed (e.g. 
Calvin Burns, Stacey Conchie, (2013) "Risk Information Source Preferences in Construction Workers", Employee Relations, Vol. 36 Iss: 1)

European Agency for Safety and Health at Work, 2013b; UK Health and Safety Executive, 2013b). The two general measures of risk (site-specific and job-specific) were included to offer a more general overview of risk information source preferences and to capture any risks that were not listed, but which may be salient to the workers.

\section{Correspondence Analysis}

Data were analysed using a correspondence analysis. This is an exploratory technique similar to principle components analysis, but for categorical data. It presents a simplified representation of the relative associations between rows and columns of a contingency table as points in a biplot. In this study, the biplot represents the distance between a source and risk, which is interpreted as the relative preference to receive information about a specific risk from a specific information source (relative to the other risks and information sources). A small distance between a source and a risk implies that workers prefer that specific source to deliver information about that specific risk more than about other risks (for a more detailed explanation of Correspondence Analysis see, Greenacre, 1993).

It should be noted that other approaches like Analytical Hierarchy Process (Saaty, 1980) could have been used in this study. This approach is common in studies of risk in complex systems, but we chose to adopt a simple ranking procedure because it easier for the workers to complete and more consistent with the approaches taken in the literature on risk information sources (e.g. Jungermann et al., 1996). Lastly, the findings from the subsequent Correspondence Analysis (i.e. biplot shown in Figure 1) allow for a more parsimonious and simpler interpretation. 
Calvin Burns, Stacey Conchie, (2013) "Risk Information Source Preferences in Construction Workers", Employee Relations, Vol. 36 Iss: 1)

\section{Results}

Table 1 shows the summed rank score for each of the five information sources for each of the eight risks. These scores are based on a sample of 101 workers as five workers were withdrawn from the analysis due to missing data. Each summed rank score was calculated by multiplying the number of participants who ranked the information source as most preferred by 5 (the score used to denote the most preferred source) and added to the number of participants who ranked the information source as second most preferred (after multiplying this number by 4), and so on. For example, the summed rank score of 345 for Back Pain and Supervisor was calculated as follows: $(22 * 5)+(28 * 4)+(29 * 3)+(14 * 2)+(8 * 1)$

\section{INSERT TABLE 1 HERE}

Table 1 shows that Supervisor was ranked as the most preferred information source, followed by Safety Manager and then Workmates, HSE, and finally Project Manager. To test if these source preferences were significantly different, RelatedSamples Wilcoxon Signed Rank Tests were conducted on the summed source ranks (a summed source rank was created for every participant by adding together the rankings for that source across the eight different risks). The results show no significant difference between Supervisor and Safety Manager, or between Workmates, HSE, and Project Manager. However, they do show significant differences between supervisors and workmates $(Z=-5.74, p<.001)$, HSE $(Z=-4.71, p<.001)$ and project manager $(Z=-$ $6.09, \mathrm{p}<.001)$. Similarly, significant differences exist between safety managers and 
Calvin Burns, Stacey Conchie, (2013) "Risk Information Source Preferences in Construction Workers", Employee Relations, Vol. 36 Iss: 1)

workmates $(Z=-4.68, p<.001)$, HSE $(Z=-4.88, p<.001)$ and project manager $(Z=-$ $6.56, \mathrm{p}<.001)$. These results suggest the existence of two distinct groups for preferred risk information source, with supervisors and safety managers being the most preferred sources to deliver risk information.

A closer inspection of the summed rank scores in Table 1 reveals some variation in this overall trend. For example, workers expressed a preference for information about asbestos from the HSE over Supervisor, and almost equal preference for information about Job-Specific Risks from Project Manager as Safety Manager. In order to investigate the pattern of information preferences, a correspondence analysis was conducted using the data in Table 1. A test of independence between the rows (i.e. risks) and columns (i.e. information sources) revealed a significant relationship; $\chi^{2}(28)=94.09$, $\mathrm{p}<.001$. The resulting biplot depicts the relationship between row (risk) and column (information source) data.

\section{INSERT FIGURE 1 HERE}

The two-dimensional biplot displayed in Figure 1 accounts for $91.02 \%$ of the inertia (i.e. total variance); the first dimension accounts for $75.46 \%$ and the second dimension accounts for $15.56 \%$ of the inertia. Along the most important first dimension (which can roughly be considered risk speciality), Safety manager and HSE are the only column points to the right of the origin, which as above suggests the existence of two distinct groups for preferred risk information source. Similarly, Heights, Back pain, and Asbestos are the only row points to the right of the origin, which suggests that these risks 
Calvin Burns, Stacey Conchie, (2013) "Risk Information Source Preferences in Construction Workers", Employee Relations, Vol. 36 Iss: 1)

are more specialised than the other risks. Along the second dimension (which can roughly be considered location-specific), Safety manager and Project manager are the only column points beneath the origin, with HSE slightly above the origin, and Workmate and Supervisor further above the origin. This suggests a division between front-line workers, the HSE (regulator), and managers as information sources. Similarly, Site transport and Site-specific risks are the only row points below the origin, which suggests a differentiation between site-specific and more generalised risks.

Further consideration of the biplot suggests a pattern of source preferences according to the nature of the risk. For example, workers show a relative preference for Supervisor and Workmates (sources with whom they presumably interact frequently) as sources of information about job-specific risks and housekeeping (everyday risks with which supervisors and workmates have experience / presumable expertise). Similarly, workers show a relative preference for Safety Manger (a source with whom they presumably interact less frequently) for site-specific risks and site transport (risks for which site safety managers have specialised knowledge). Lastly, workers express a relative preference for the HSE (a source with which they presumably interact less frequently still) for information about asbestos and back pain (risks about which the HSE has specialised knowledge but which may be perceived as more risky due to higher levels of uncertainty and unknown variables). The results suggest that workers' source preferences are not generic (i.e. workers choose their preferred source based on the risk being considered) and that source expertise is more important than source proximity in determining the preferred source of occupational risk information. 
Calvin Burns, Stacey Conchie, (2013) "Risk Information Source Preferences in Construction Workers", Employee Relations, Vol. 36 Iss: 1)

\section{Discussion}

The aim of this study was to investigate risk information source preferences in construction workers. Specifically, we investigated whether occupational risk information source preference was risk independent. In general, construction workers rated supervisors and safety managers as their most preferred sources of risk information. A correspondence analysis though suggested that workers' risk information source preference is risk dependent and might be driven by source expertise. For example, for information about asbestos, workers expressed a preference for information from the UK HSE. Essentially, workers expressed a preference to receive information from those sources they regard as having the most expertise, or experience, with a particular risk.

The suggestion that source expertise is important in decisions regarding source preferences is consistent with previous research on the general public's perceptions of industrial / technological hazards and risks. For example, Jungermann et al. (1996) found that laypeople preferred to receive different types of information about the risks of a chemical plant from different sources (e.g., the company was the preferred information source about what is produced and which products are manufactured, but environmental groups were the preferred source for information about health risks and potential accidents). Our findings show that this effect is consistent even when the relative rank position of a source is considered. Unlike Jungermann et al. (1996), we examined how workers ranked a source in terms of how much that source was preferred relative to other sources, instead of just focusing on the most preferred source.

Our findings have important practical implications for the role of safety managers in risk communication. With the exception of Dingsdag et al. (2008), very few studies 
have focused on the role of safety managers in shaping workers' safety behaviours. Previously, we found that a similar sample of construction workers rated the HSE and safety managers as the most trusted information sources for the same risks ${ }^{2}$ and that they were the most influential sources in shaping construction workers' risk-related behavioural intentions (Conchie \& Burns, 2009). In this study, we found that supervisors and safety managers were overall the most preferred sources of risk information. Given that safety managers are one of the most trusted information sources (and that trust in an information source is risk independent; see Conchie \& Burns, 2009), they may be able to increase their influence on workers' risk-related behavioural intentions by consulting workers' preferred risk-dependent sources of information (e.g. the HSE for asbestos) and citing that source when they communicate with workers about that risk. Although the full extent of safety managers' influence is currently unknown, we expect them to have multiple influences on workers' safety behaviours, which in some situations, will be at least comparable to that documented from supervisors and an organisation's safety climate (e.g. Mearns \& Reader, 2008; Melià et al., 2008).

Our findings also have implications for trust building within high-hazard organisations. The leading model of trust in organisations (Mayer et al., 1995) suggests that a person's perceived trustworthiness is based on perceptions of ability, benevolence, and integrity. Trust is widely acknowledged to be domain-specific and with respect to occupational safety, Conchie et al. (2006) found that expectations about a person's safety-related behaviours and safety-related intentions are important determinants of trust. Previously, we found that construction workers' trust in risk information from the

\footnotetext{
${ }^{2}$ Conchie and Burns (2009) only investigated four risks (back pain, heights, slips/trips, and site transport) but these four risks were among the eight risks investigated in this study.
} 
Calvin Burns, Stacey Conchie, (2013) "Risk Information Source Preferences in Construction Workers", Employee Relations, Vol. 36 Iss: 1)

project manager, safety manager, HSE, and workmates was based on the source's accuracy (Conchie \& Burns, 2009). Given our current findings that construction workers appear to prioritize source expertise in determining information source preference, it may be possible to build trust with respect to safety in project managers, safety managers, and workmates by encouraging them to talk about the risks for which they are the preferred information sources more frequently. For example, if workers talk about housekeeping (a risk for which they are the preferred source of information) more frequently during safety meetings or shift briefings, this may allow them opportunities to also demonstrate their care and concern for their fellow workers' safety (i.e. benevolence) and their safetyrelated behavioural intentions (i.e. integrity). This may lead to an increase in trust for those workers through more positive perceptions of their benevolence and integrity, which as per Mayer et al. (1995) are important factors of trustworthiness. This may also lead to a more positive safety culture within the organisation as trust is the foundation of an effective safety culture (Burns, Mearns, \& McGeorge, 2006).

This study has provided an important first step in investigating risk information source preferences in an occupational setting. It is still unclear though whether workers who communicate with their preferred source of risk information form more accurate perceptions of risk than workers who communicate with a non-preferred source. It is also unclear whether workers who learn through experience or through passive risk communication form more accurate perceptions of risk than workers who actively seek out risk information. Research in non-industrial areas has taken a slightly different approach and focused on risk perceptions as they relate to internal and external searching behaviours (e.g., Engel et al., 1999; Hawkins et al., 1998). 
Calvin Burns, Stacey Conchie, (2013) "Risk Information Source Preferences in Construction Workers", Employee Relations, Vol. 36 Iss: 1)

Internal searching refers to situations in which an individual uses memory or recall (e.g., experience or vicarious reinforcement). The safety literature makes reference to types of internal searches in which workers may engage. For example, studies have shown that individuals do not engage with published material about risk or safety warnings if they consider themselves to be experienced (Duijne et al., 2008; Lehto \& Foley, 1991; Wright et al., 1982). In these cases, individuals are more likely to draw on their own experiences and rely on internal search strategies for estimating risk. This may lead though to biased perceptions of risk through melioration bias (underestimating the likelihood of a negative event occurring to oneself), rare-event bias (underestimating the occurrence of a low-frequency event), and optimism bias (the tendency to perceive others as being at greater risk than oneself).

External searching refers to an individual's purposeful behaviour that seeks to collect information about risk from an external source. Although our decision to use human sources in the current study was an informed one, the importance of non-human sources (e.g. the internet, or articles in newspapers or trade magazines) should not be overlooked and should be considered in future work. Dwyer (1991) noted that multiple subcontractors working laterally and vertically creates problems for construction safety systems like who is responsible for workers' safety and how a single system can be implemented in a fragmented climate. This lack of clarity is strengthened by the fact that smaller subcontractor companies name the individual worker as his / her own safety officer (Eakins, 1992; Holmes \& Gifford, 1997), which may be quite different to the procedures operated by larger companies. In these climates, interpersonal relations may 
Calvin Burns, Stacey Conchie, (2013) "Risk Information Source Preferences in Construction Workers", Employee Relations, Vol. 36 Iss: 1)

be distant, impersonal and possibly hostile. Under these conditions, workers may find non-human sources of information more useful.

Future research should also investigate how risk perceptions change as a result of new information from preferred and non-preferred information sources. Risk perception is analogous to a Bayesian decision process (Liu et al., 1998). In a Bayesian decision process, an individual's risk perception is assumed to vary with the information at any given time. A person's risk perception depends on weightings of prior beliefs and new information as it becomes available. Studies of occupational risk (e.g., chemicals; Viscusi \& O’Connor, 1984) and environmental risk (e.g., radon; Smith \& Johnson, 1988) support modified forms of Bayesian learning models to describe how individuals use information to revise their risk perceptions. Investigating the role of new information from a preferred information source in a Bayesian learning model versus the role of information from a non-preferred information source would have important implications for educating workers about risk.

While this study has provided an important first step in investigating risk information source preferences in an occupational setting, it is not without limitations. One limitation is that the sample was recruited from a single building site, which may limit the generalizability of the results. To mitigate this, the ranking exercise focused on information sources available to construction workers on most types of construction sites (e.g., buildings, roads) and on risks to which construction workers are exposed globally. Thus, we expect that our results are representative of construction workers in general. The extent to which our findings are representative of how workers prioritize risk 
Calvin Burns, Stacey Conchie, (2013) "Risk Information Source Preferences in Construction Workers", Employee Relations, Vol. 36 Iss: 1)

information source preference in other industries is unclear and should be explored in future research. 
Calvin Burns, Stacey Conchie, (2013) "Risk Information Source Preferences in Construction Workers", Employee Relations, Vol. 36 Iss: 1)

\section{References}

Aronsson, G. (1999). Contingent workers and health and safety. Work, Employment \& Society, 13(3), 439-459.

Burns, C., Mearns, K., \& McGeorge, P. (2006). Explicit and implicit trust within safety culture. Risk Analysis, 26, 1139-1150.

Burt, C., Chmiel, N., \& Hayes, P. (2008). Implications of turnover and trust for safety attitudes and behaviour in work teams. Safety Science, 47, 1002-1006.

Conchie, S.M., \& Burns, C. (2009). Improving occupational safety: Using a trusted information source to communicate about risk. Journal of Risk Research, 12(1), $13-25$.

Conchie, S.M., I.J. Donald, and P.J. Taylor. (2006). Trust: Missing piece(s) in the safety puzzle. Risk Analysis, 26, 1097-1104.

Cox, S. and Cox, T., (1991). The structure of employee attitudes to safety: A European example. Work and Stress, 5, 93-106.

Dingsdag, D. P., Biggs, H. C. and Sheahan, V. L. (2008). Understanding and defining OH\&S competency for construction site positions: Worker perceptions. Safety Science, 46, 619-633.

Duijne, F. H., Aken D. V., \& Schouten, E. G. (2008). Considerations in developing complete and quantified methods for risk assessment. Safety Science, 46, 245-254.

Dweyer, T. (1991). Life and death at work: Industrial accidents as a case of socially produced error. Plenum, New York.

Eakins, J. (1992). Leaving it up to the workers: Sociological perspectives on the management of health and safety in small workplaces. International Journal of Health Services, 22, 689-704.

Engel, J. F., Blackwell, R. D., \& Miniard, P. W. (1999). Consumer behaviour (5 ${ }^{\text {th }}$ ed.). Dryden Press, Chicago.

European Agency for Safety and Health at Work (2013a). Construction. Available at http://osha.europa.eu/en/sector/construction (accessed 1 March 2013).

European Agency for Safety and Health at Work (2013b). Tackling major hazards and risks. Available at http://osha.europa.eu/en/sector/construction/index_html/hazards_risks (accessed 1 March 2013) 
Frewer, L. J., Howard, C., Hedderley, D. and Shepherd, R. (1996). What determines trust in information about food-related risks? Underlying psychological constructs. Risk Analysis, 16, 473-485.

Greenacre, M. J. (1993). Correspondence analysis in practice. Academic Press; London.

Hawkins, D. I., Best, R. J., \& Coney, K. A. (1998). Consumer behaviour: Implications for marketing strategy $\left(7^{\text {th }}\right.$ Ed.). Irwin Inc, Chicago.

Hayes, B. E., Perander, J., Smecko, T. and Trask, J. (1998). Measuring perceptions of workplace safety: Development and validation of the work safety scale. Journal of Safety Research, 29, 145-161.

Helmreich, R. L., \& Merritt, A.C. (1998). Culture at work in aviation and medicine: National, organizational and professional influences. Ashgate: Aldershot.

Holmes, N., \& Gifford, S. M. (1997). Narratives of risk in occupational health and safety: Why the 'good' boss blames his tradesmen and the 'good' tradesman blames his tools. Australian and New Zealand Journal of Public Health, 21, 11-19.

Johnson, J. D. (1996). Information seeking: An organizational dilemma. Westport, CN: Quorum Books.

Jungermann, H., Pfister, H. R. and Fischer, K. (1996). Credibility, information preferences and information interests. Risk Analysis, 16, 251-261.

Lehto, M. R., \& Foley, J. P. (1991). Risk-taking, warning labels, training, and regulation: Are they associated with the use of helmets by all terrain vehicle riders? Journal of Safety Research, 22, 191-200.

Lingard, H. and Rowlinson, S. (2005). Occupational Health and Safety in Construction Project Management. Spon Press, New York.

Liu, S., Huang, J., \& Brown, G.L. (1998). Information and risk perception: A dynamic adjustment process. Risk Analysis, 18, 689-699.

Mayer, R. C., Davis, J. H., \& Schoorman, F. D. (1995). An integrative model of organizational trust. Academy of Management Review, 20, 709-734.

Mearns, K., \& Reader, T. (2008). Organizational support and safety outcomes: An uninvestigated relationship? Safety Science, 46, 388-397.

Mearns, K., \& Yule, S. (2009). The role of national culture in determining safety performance: Challenges for the global oil and gas industry. Safety Science, 47, 777-785. 
Meliá, J. L., Mearns, K., Silva, S. A. and Lima, L. (2008). Safety climate responses and the perceived risk of accidents in the construction industry. Safety Science, 46, 949958.

Powell, C. (2007). The perception of risk and risk taking behavior: Implications for incident prevention strategies. Wilderness and Environmental Medicine, 18, 10-15.

Rousseau, D. M., Sitkin, S. B., Burt, R. S., \& Camerer, C. (1998). Not so different after all: A cross-discipline view of trust. Academy of Management Review, 23, 393404.

Saaty, T.L., (1980). The analytical hierarchy process: Planning, priority setting resource allocation. McGraw-Hill: New York.

Savolainen, R. (2008). Everyday information practices: A social phenomenological perspective. Lanham, Maryland: The Scarecrow Press.

Seo, D. C., Torabi, M. R., Blair, E. H. and Ellis, N. T., (2004). A cross validation of safety climate scale using confirmatory factor analytic approach. Journal of Safety Research, 35, 427-445.

Sinclair, A., Hill, D. and Tyers, C. (2008). Irish and Non-Irish National Construction Workers: Research on differences in approach to health and safety at work. Health and Safety Authority (Ireland).

Smith, V.K., \& Johnson, F.R. (1988). How do risk perceptions respond to information? The case of radon. The Review of Economics and Statistics, 70, 1-8.

Spangenberg, S., Baarts, C., Dyreborg, J., Jensen, L., Kines, P., \& Mikkelsen, K.L. (2003). Factors contributing to the difference in work related injury rates between Danish and Swedish construction workers. Safety Science, 41(6), 517-530.

UK Health \& Safety Executive (2013a). Health and safety in the construction industry. Available at http://www.hse.gov.uk/construction/index.htm (accessed 1 March 2013).

UK Health and Safety Executive. (2013b). The High 5. Available at http://www.hse.gov.uk/pubns/indg384.pdf (accessed 1 March 2013).

US Department of Labor, Bureau of Labor Statistics (2013). Census of Fatal Occupational Injuries Summary, 2011. Available at http://www.bls.gov/news.release/cfoi.nr0.htm (accessed 1 March 2013). 
Viscusi, W.K., \& O’Connor, C.J. (1984). Adaptive responses to chemical labeling: Are workers Bayesian decision makers? The American Economic Review, 74, 942956.

Warner, E. S., Murray, A. D. and Palmour, V. E. (1973). Information needs of urban residents. Washington, DC.

Wright, P., Creighton, P., \& Threlsall, S. M. (1982). Some factors determining when instructions will be read. Ergonomics, 25, 225-237.

Zohar, D. and Luria, G. (2004). Climate as a Social-cognitive Construction of Supervisory Safety Practices: Scripts as Proxy of Behavior Patterns, Journal of Applied Psychology, 89, 322-333. 
Calvin Burns, Stacey Conchie, (2013) "Risk Information Source Preferences in Construction Workers", Employee Relations, Vol. 36 Iss: 1)

Table 1: Summed rank score of preferred risk information source for eight risks

Supervisor Safety Manager Workmates HSE $\quad$ Project Manager

$\begin{array}{rllllll}\text { Asbestos } & 325 & 356 & 254 & 344 & 236 \\ \text { Back Pain } & 345 & 366 & 277 & 297 & 230 & 276 \\ \text { Site Transport } & 354 & 381 & 246 & 268 & 258 & 236 \\ \text { Heights } & 370 & 352 & 279 & 250 & 264 \\ \text { Slips / Trips } & 358 & 364 & 293 & 231 & 254 & 307 \\ \text { Housekeeping } & 394 & 343 & 286 & 213 & 276 \\ \text { Job-Specific Risks } & 399 & 310 & 249 & & \mathbf{2 1 3 3} & \mathbf{2 0 7 9} \\ \text { Site-Specific Risks } & 359 & 380 & \mathbf{2 1 5 2} & \end{array}$


Figure 1: Two-dimensional plot of Source x Risk correspondence analysis of employees' ranked source preferences

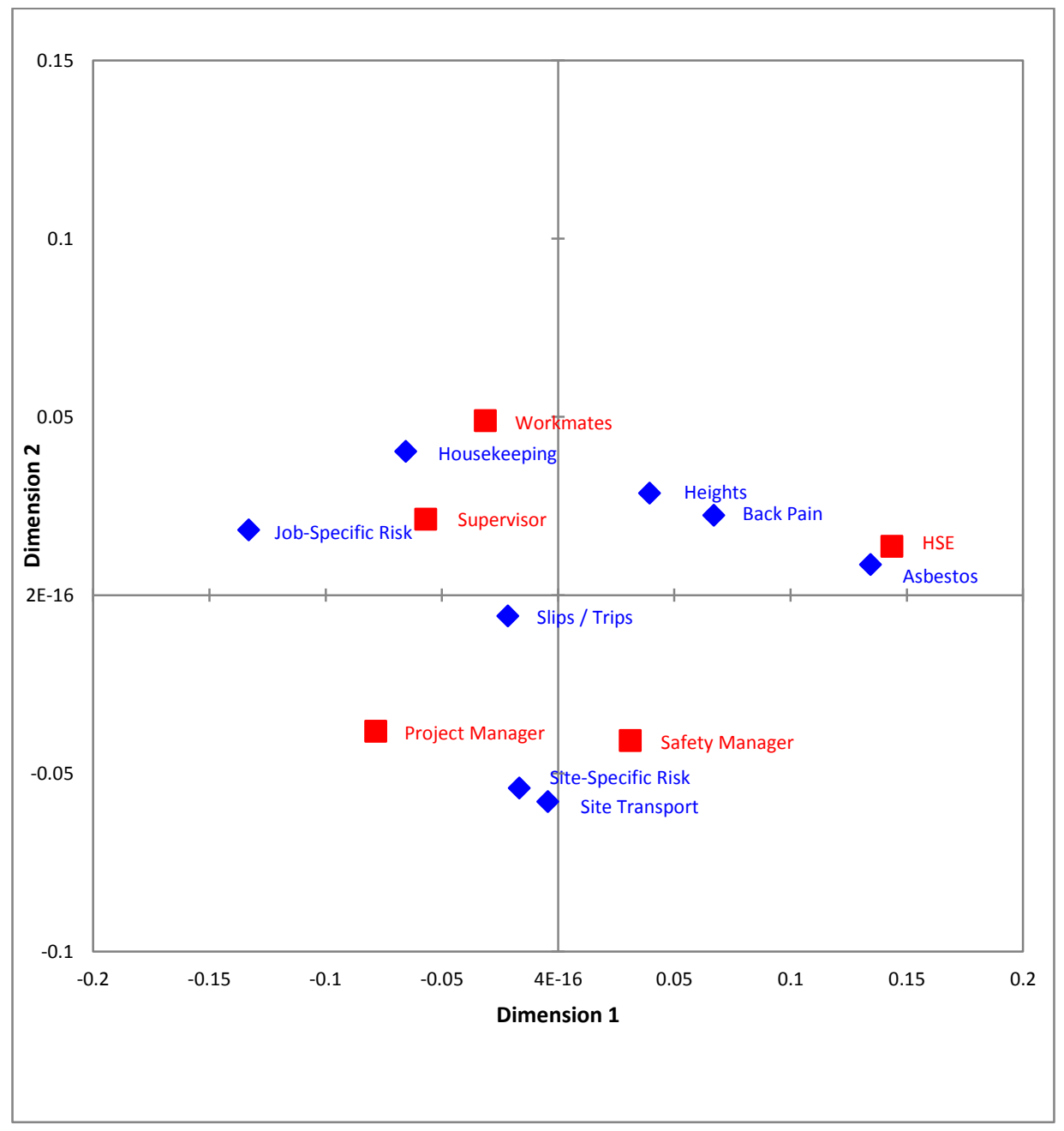

